\title{
Metastasiertes kolorektales Karzinom
}

\section{Individualisierte Therapie mit Bevacizumab plus Chemotherapie}

Aktuelle Studiendaten, die auf dem diesjährigen ESMO/ECCO vorgestellt wurden, belegen erneut das breite Anwendungsspektrum des VEGF (Vascular Endothelial Growth Factor)-Hemmers Bevacizumab $\left(\right.$ Avastin $\left.^{\circledR}\right)$ beim metastasierten kolorektalen Karzinom ( $m C R C)$. Sowohl Patienten, für die eine weniger aktive Therapie indiziert ist, als auch solche, die die effektivste jeweils verfügbare systemische Kombinationstherapie erhalten sollten, profitieren von dem VEGFHemmer. Durch die Wahl des in der jeweiligen klinischen Situation am besten geeigneten Chemotherapiepartners lässt sich die Intensität der Bevacizumab-haltigen Behandlung dem individualisierten Therapieziel anpassen.

Die Phase-III-Studie AGITG-MAX untersuchte die Kombination von Capecitabin (C) vs. Capecitabin/Bevacizumab (CB) vs. Capecitabin/Bevacizumab/Mitomycin $\mathrm{C}$ (CBM) als potenziell niedrig-toxische Regime für Patienten, die nicht für eine Oxaliplatin- oder Irinotecan-basierte Therapie in Frage kommen [1]. In die Studie wurden 471 Patienten mit einem medianen Alter von 67 Jahren eingeschlossen. 30\% der Patienten sprachen auf C, 38\% auf CB und $46 \%$ in der Kombination CBM an (C vs. CBM: $p=0,006$ ). Das progressionsfreie Überleben (PFS) war in beiden Bevacizumab-Armen signifikant höher als unter der Capecitabin-Monotherapie (5,7 vs. 8,5 vs. 8,4 Monate; $\mathrm{C}$ vs. CB: $\mathrm{p}<0,001$; $\mathrm{C}$ vs. CBM: $\mathrm{p}<0,001$ ) (Abb. 1). Das Gesamtüberleben (OS) in den $3 \mathrm{Be}$ handlungsarmen war nicht signifikant verschie- den (18,9 vs. 18,9 vs. 16,4 Monate). Da rund zwei Drittel der Patienten nach Tumorprogress eine weitere systemische Therapie erhielten, sind die OS-Daten nach Aussage der Studienautoren jedoch nicht aussagekräftig in Bezug auf die First-line-Therapie. Die Bevacizumabhaltige Therapie war gut verträglich - lediglich das Hand-Fuß-Syndrom (Grad 3-5: 16 vs. 26 vs. $28 \%$ ) und arterielle Thromboembolien (Grad 3-5: 0,0 vs. 2,5 vs. 3,8\%) traten unter der Kombination mit Bevacizumab signifikant häufiger auf als unter der Capecitabin-Monotherapie.

\section{Gut verträgliche Regime auch für ältere Patienten}

Den Nutzen von Bevacizumab für ein älteres Patientenkollektiv bestätigt eine Subgruppenanalyse der Beobachtungsstudie First BEAT, in der die Sicherheit und Wirksamkeit von Bevacizumab in Kombination mit gängigen Chemotherapien in der täglichen Praxis untersucht wurden. Eine Datenanalyse in Bezug auf das Alter der 1914 Patienten ( $<65$ Jahre, 65-74 Jahre, $\geq 75$ Jahre) zeigte keinen Unterschied im PFS [2]. Das OS war, wie erwartet, bei den über 75-Jährigen etwas geringer als bei den Jüngeren. Die Nebenwirkungen waren bei allen Altersgruppen vergleichbar, ausgenommen leicht erhöhter Raten von thromboembolischen Ereignissen und Proteinuria bei den über 75-Jährigen. Damit werden die Daten der BRiTEStudie [3] sowie der bisherigen Phase-III-Studien hinsichtlich des Outcomes der älteren Patienten unter Bevacizumab bestätigt.

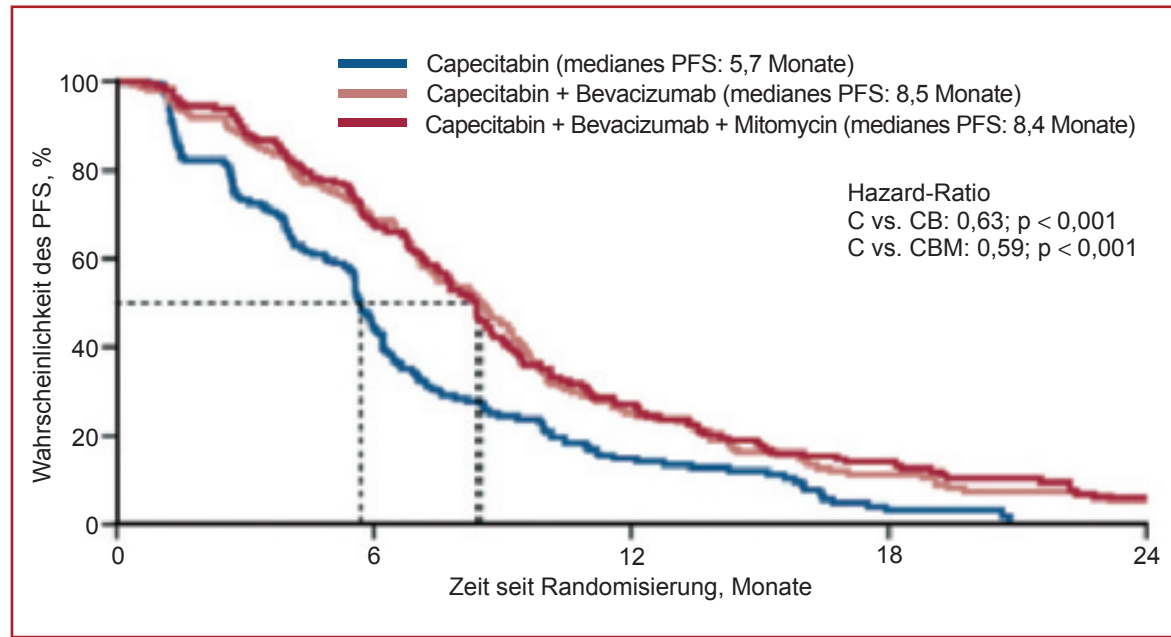

Abb. 1. Progressionsfreies Überleben - AGITG-MAX-Studie: Capecitabin-Monotherapie vs. Kombination mit Bevacizumab, modifiziert nach [1].

\section{Prospektive Daten zur sekundären Metastasenresektion}

Neue Evidenz für den Nutzen einer Bevacizumab-haltigen Therapie bei Patienten mit primär irresektablen Lebermetastasen lieferte die prospektive einarmige Phase-II-Studie BOXER [4]. Es wurden 45 Patienten mit nicht resektablen oder zeitgleich mit dem Primärtumor diagnostizierten Lebermetastasen neoadjuvant mit Bevacizumab plus XELOX behandelt. Das Ansprechen betrug 78\%. Zur Zeit der Auswertung konnten rund 36\% der Patienten einer Leberresektion zugeführt werden (R0 56\%; R1 31\%; R2 13\%). Die Resektabilität wurde alle 4 Zyklen überprüft. Das Regime wurde gut vertragen, es traten keine unerwarteten Nebenwirkungen auf und es wurden keine perioperativen Komplikationen beobachtet. Die Ergebnisse stärken damit die bereits publizierte Evidenz [5-8] für den sicheren Einsatz von Bevacizumab bei mCRC-Patienten mit primär irresektablen Lebermetastasen und dem Ziel der sekundären Metastasenresektion.

\section{Referenzen}

Tebbutt et al.: ESMO 2009; abstract 6001, ora presentation.

2 Van Cutsem et al.: ESMO 2009; abstract 6088, poster presentation.

Grothey et al.: JCO 2008;26:5326-34.

4 Wong et al.: ESMO 2009; abstract 6076, poster presentation.

presentation.
Cassidy et al.: ASCO 2008; poster 4022.

5 Cassidy et al.: ASCO 2008; poster 4022.

Van Cutsem et al.: Annal Oncol 2009;20:1842-7.

8 Gruenberger et al.: J Clin Oncol 2008;26:1830-1835.

Impressum

Metastasiertes kolorektales Karzinom

Individualisierte Therapie mit Bevacizumab plus Chemotherapie

PharmaForum in ONKOLOGIE 32 | 12 | 09

C 2009 by S. Karger Verlag für Medizin und Naturwissenschaften $\mathrm{GmbH}$

Wilhelmstraße 20A

79098 Freiburg, Deutschland

Mit freundlicher Unterstützung durch Roche Pharma AG.

Der Verlag und die Herausgeber der Zeitschrift übernehmen keine Verantwortung für diese Rubrik. 


\section{Fortgeschrittenes Nierenzellkarzinom}

\section{mTOR-Inhibitor Everolimus schließt wesentliche Therapielücke}

\section{Mit dem oral verfügbaren mTOR-Inhibitor Everolimus (Afinitor ${ }^{\circledR}$ ) steht seit August 2009 \\ Everolimus nach einer TKI-Therapie erfolgreich} eine Therapie für Patienten mit fortgeschrittenem Nierenzellkarzinom zur Verfügung, bei denen es während oder nach einer Behandlung mit einem Tyrosinkinase-Inhibitor zur Tumorprogression gekommen ist. Zur Zulassungsstudie RECORD-1 wurden 2 ergänzende Auswertungen mittels statistischer Verfahren durchgeführt, die den Effekt des Crossovers von Patienten aus der Placebo- in die Verumgruppe berïcksichtigen. Diese weisen darauf hin, dass sich die Verlängerung des progressionsfreien Überlebens unter Everolimus auch in einem verlängerten Gesamtüberleben wiederspiegelt, wie Prof. Lothar Bergmann, Medizinische Klinik II der Universität Frankfurt/Main, anlässlich des DÖSGHO 2009 in Mannheim berichtete.

Das mammalian Target Of Rapamycin (mTOR) nimmt eine Schlüsselposition im Signaltransduktionsweg ein, der von entscheidender Bedeutung für Wachstum, Teilung und Stoffwechsel maligner Zellen ist. Außerdem steigert dieses Enzym durch vermehrte Ausschüttung von Wachstumsfaktoren indirekt die Tumor-Angiogenese. Bei vielen Tumorerkrankungen, so auch beim Nierenzellkarzinom (RCC), findet eine Überaktivierung von mTOR statt. Damit verbunden ist einerseits eine unkontrollierte Zellproliferation, andererseits eine Neubildung von Gefäßen, die der Tumor zum Wachstum benötigt. Da mit Everolimus Tumor-Zellwachstum sowie Tumor-Angiogenese gehemmt werden, besitzt dieser mTOR-Inhibitor einen dualen Wirkmechanismus.

Jährlich erkranken in Deutschland den Statistiken des Robert Koch-Institutes zufolge circa 14500 Menschen an einem RCC [1]. Etwa ein Drittel weist bei der Diagnose bereits Metastasen auf, berichtete Prof. Bergmann. In Deutschland sind Tyrosinkinase-Inhibitoren (TKI), die zu den gegen den VascularEndothelial Growth Factor (VEGF) gerichteten Therapien zählen, inzwischen Standard in der Behandlung des fortgeschrittenen Nierenzellkarzinoms. Ungeklärt war bislang jedoch, welche Therapie anzuwenden ist, wenn die Tumorerkrankung während oder nach einer Behandlung mit dem ersten TKI fortschreitet. Die Umstellung auf einen weiteren TKI kann die Resistenz nur partiell überwinden, erinnerte Prof. Bergmann.
Die placebokontrollierte, doppelblinde PhaseIII-Studie RECORD(Renal Cell Cancer Treatment With Oral RAD001 Given Daily)-1 sollte klären, ob Everolimus in dieser Situation einen klinischen Nutzen zeigt [2]. Dazu wurden 416 Patienten mit fortgeschrittenem RCC, die zuvor mindestens einen TKI erhalten hatten, im Verhältnis 2:1 randomisiert. Die Betroffenen wurden mit einer Tablette $10 \mathrm{mg} / \mathrm{d}$ Everolimus oder Placebo jeweils in Kombination mit «Best Supportive Care» behandelt. Primärer Endpunkt war das progressionsfreie Überleben. Patienten aus der Placebogruppe, bei denen es zum Progress kam, hatten die Möglichkeit, eine unverblindete Behandlung mit Everolimus zu erhalten. Diese Tatsache ist wichtig für die Bewertung der Resultate zum medianen Gesamtüberleben einem sekundären Endpunkt dieser Studie [3]. Nach einer vorab geplanten Interimsanalyse, in der Everolimus eine signifikant überlegene Wirksamkeit zeigte, wurde die Studie vorzeitig beendet. Der oral verfügbare mTOR-Inhibitor verlängerte das progressionsfreie Überleben deutlich auf mehr als das Doppelte von 1,9 auf 4,9 Monate ( $<<0,001$; HR 0,33; 95\%-CI 0,250,43). Nach 10 Monaten Everolimus-Therapie waren Patienten mit einer Wahrscheinlichkeit von mehr als $25 \%$ progressionsfrei. Zugleich reduzierte Everolimus signifikant das Risiko von Nierenzellkarzinom [4].
Krankheitsprogression oder Tod um 67\%. Bei 69\% der Patienten wurde ein klinischer Nutzen unter der Behandlung mit dem oral verfügbaren mTOR-Inhibitor registriert (Placebo: 32\%), davon bei $67 \%$ eine Tumorstabilisierung und bei $2 \%$ eine partielle Remission. Patienten aller Subgruppen, unabhängig von Alter, Geschlecht, Risikoklassifizierung und Vorbehandlung, profitierten von der Behandlung mit Everolimus. Damit ist die Therapie mit diesem mTORInhibitor die einzige, für die eine Wirksamkeit beim fortgeschrittenen RCC nach TKI-Versagen in einer Phase-III-Studie nachgewiesen werden konnte, so Prof. Bergmann.

Beim medianen Gesamtüberleben als sekundärem Endpunkt ergab die ITT(Intent-to-treat)Analyse keinen signifikanten Unterschied zwischen Verum- und Placeboarm (14,8 versus 14,4 Monate; $p=0,177$ ) [3]. Die Tatsache, dass insgesamt 112 von 139 Patienten (81\%) aus der Placebogruppe nach Progress oder nach Entblindung Everolimus erhielten, schränkt die Aussagekraft dieser Auswertung jedoch erheblich ein. Aus diesem Grund wurden 2 weitere, statistische Erhebungen durchgeführt: Zum einen die RPSFT (Rank Preserving Structural Failure Time)-Analyse, die es erlaubt, die zusätzliche Überlebenszeit abzuschätzen, die ein Patient durch eine Everolimus-Therapie gewinnt (d.h. sowohl bei Patienten in der ursprünglich randomisierten Verumgruppe als auch bei Patienten nach Crossover vom Placebo- in den Verumarm).

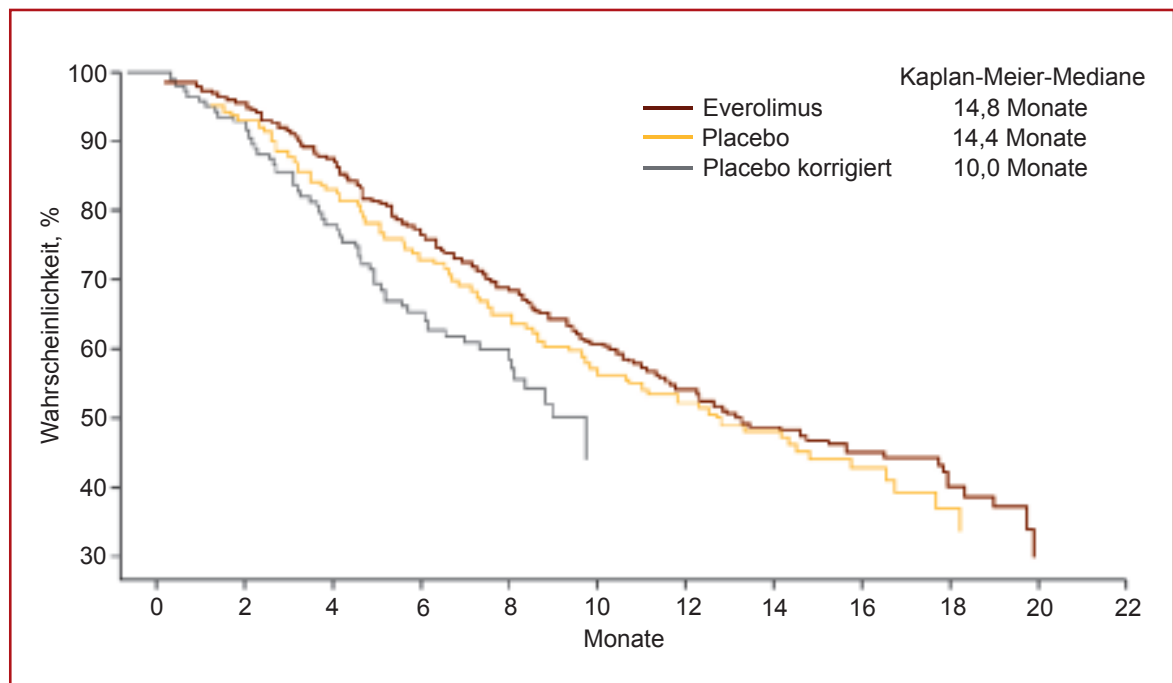

Abb. 1. RPSFT-Analyse zur RECORD-1-Studie: Medianes Gesamtüberleben beim fortgeschrittenen 
Die RPSFT-Analyse ergab, dass Patienten bei einer Therapie mit diesem mTOR-Inhibitor eine 1,9-fach längere (95\%-CI $0,5-8,5)$ relative Überlebenszeit im Vergleich zu einer PlaceboBehandlung haben (Abb. 1). Die berechnete mediane Überlebenszeit ohne Crossover-Effekt ergab für die Placebogruppe 10,0 Monate im Vergleich zu den nicht um Crossover korrigierten 14,4 Monaten bei der ITT-Analyse [4]. Zum anderen wurde eine Erhebung mittels IPCW (Inverse Probability of Censoring Weights)Coxmodel durchgeführt. In dieser konnte nachgewiesen werden, dass Everolimus das Risiko der Mortalität um 45\% reduziert (HR 0,55; 95\%-CI 0,31-0,97) [5]. Beide Auswertungen weisen darauf hin, dass sich die signifikante Verlängerung des progressionsfreien Überlebens unter Everolimus auch in eine deutliche Verlängerung des Gesamtüberlebens überträgt.

Die statistischen Untersuchungen RPSFT und IPCW Coxmodel werden standardmäßig immer dann verwendet, wenn in einer Studie der Wechsel von Patienten aus dem Placebo- in den Wirkstoffarm möglich ist und der Crossover-Effekt korrigiert werden soll. So wurden diese statistischen Methoden angewandt im Rahmen der Auswertung der Studie zur Zulassung von Sunitinib bei gastrointestinalen Stromatumoren [6, 7].

Everolimus hat ein günstiges Nebenwirkungsprofil. Die unerwünschten Wirkungen waren meist mild. Grad-3/4-Toxizitäten traten nur vereinzelt auf, betonte Prof. Bergmann. Entsprechend gering blieb mit 7\% die Abbruchrate aufgrund therapiebedingter, unerwünschter Wirkungen.

Die Lebensqualität verschlechterte sich durch Everolimus im Vergleich zu Placebo nicht. Vielmehr blieb der Karnofsky Performance Status unter diesem mTOR-Inhibitor signifikant länger stabil als unter Placebo (5,8 Monate versus 3,8 Monate; $p=0,004)$, was den Gewinn an Lebensqualität für den Patienten unter einer Everolimus-Therapie weiterhin unterstreicht [3].

\section{Leitlinien berücksichtigen neue Erkenntnisse bereits}

Auf Basis der Ergebnisse von RECORD-1 empfehlen die internationalen Leitlinien Everolimus als Therapiestandard zur Behandlung des fortgeschrittenen RCC nach TKI. Die Leitlinie des National Comprehensive Cancer Network (NCCN) benennt diesen mTOR-Inhibitor mit einer Kategorie-1-Empfehlung als bevorzugte Therapieoption nach einem TKI [8] (Abb. 2). Gleiches gilt sowohl für die European Organisation for Research and Treatment of CancerGenitourinary als auch für die European Association of Urology: Bereits vor der Zulassung wurde dieser oral verfügbare mTOR-Inhibitor nach einer TKI-Therapie mit einer Level-1bbzw. Grad-A-Empfehlung aufgenommen $[9,10]$.

\section{Umfassendes Studienprogramm prüft weitere Optionen}

Das RECORD-Programm wird mit den Studien RECORD-2 und -3 fortgesetzt. Die RECORD2-Studie untersucht die Möglichkeit, Everolimus in der Erstlinientherapie des fortgeschrittenen RCC mit dem VEGF-Antikörper Bevacizumab zu kombinieren, gegenüber der Behandlung mit Bevacizumab plus Interferon $\alpha-2 a$. In diese sollen 360 Patienten eingeschlossen werden. Hierfür läuft die Rekrutierung seit August 2009.

Die RECORD-3-Studie vergleicht den oral verfügbaren mTOR-Inhibitor in der Erstlinienthera-

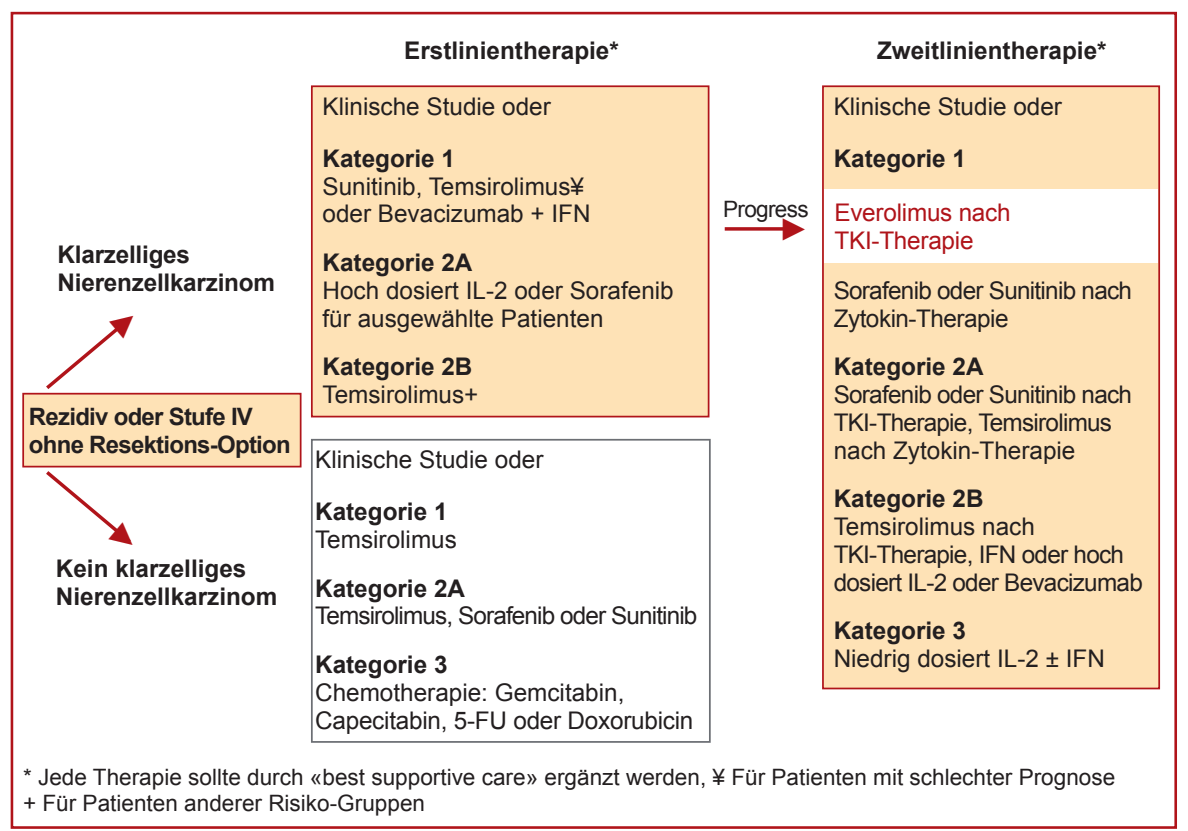

Abb. 2. Übersicht der Guidelines des NCCN, modifiziert nach [8]. pie des fortgeschrittenen RCC mit dem TKI Sunitinib, der derzeit als Standard in dieser Indikation gilt. Es ist geplant, 390 Patienten zu rekrutieren. Mit der RAPTOR(RAD001 in Advanced Papillary Tumor Program in Europe)-Studie wird untersucht, ob auch Patienten mit papillärem RCC von einer Behandlung mit Everolimus profitieren können. Wie Prof. Bergmann sagte, geben Kohortendaten aus anderen Studien Hinweise, dass diese Vermutung zutreffen könnte. RAPTOR ist als einarmige Multicenterstudie mit 60 Patienten geplant, die an einem fortgeschrittenen, papillären RCC leiden und noch keine systemische Vorbehandlung erhalten haben.

Der Wirkmechanismus des oral verfügbaren mTOR-Inhibitors lässt erwarten, dass nicht nur das Nierenzellkarzinom, sondern auch andere Tumorentitäten auf Everolimus ansprechen werden. Deshalb wird dieser mTOR-Inhibitor im Rahmen des Studienprogramms WIDE (Worldwide Initiative to Develop Everolimus) bei weiteren Krebserkrankungen erprobt, unter anderem bei neuroendokrinen Tumoren, Brust-, Leberund Magenkrebs sowie malignen Lymphomen.

Manuela Arand, Berlin

\section{Quelle}

Satellitensymposium «Update zur Therapie solider Tumoren heute!» bei der gemeinsamen Jahrestagung der Deutschen, Österreichischen und Schweizerischen Gesellschaften für Hämatologie und Onkologie, Mannheim, 3. Oktober 2009, Veranstalter: Novartis Pharma GmbH

\section{Referenzen}

1 Robert Koch-Institut und die Gesellschaft der epidemiologischen Krebsregister in Deutschland e.V. (Hrsg): Krebs in Deutschland 2003-2004. Häufigkeiten und Trends. ed 6. Berlin, 2008

2 Motzer RJ et al.: The Lancet 2008;372:449-456.

3 Motzer RJ et al.: Genitourinary Cancers Symposium, Orlando 2009; Poster und Kay A et al.: Genitourinary Cancers Symposium, Orlando 2009; abstr 278.

4 Korhonen M et al.: ESMO/ECCO, Berlin 2009; European Journal of Cancer Supplements 2009;7 abstr 7155 .

5 Wiederkehr D et. al.: ESMO/ECCO, Berlin 2009; European Journal of Cancer Supplements 2009;7 abstr 7131 und Poster.

6 Demetri GD et al.: J Clin Oncol 2008;26:559, abstr 10524

7 Schöffski P et al.: ESMO, Stockholm 2008; abstr 8650.

8 NCCN Clinical Practice Guidelines in Oncology, Kidney Cancer: Natl Compr Canc Netw 2009;7:618-630.

9 De Reijke M et al.: Eur J Cancer 2009;45:765-773.

10 Ljungberg B et al.: Guidelines on renal cell carcinoma, EAU 2009;23.

\section{Impressum}

Fortgeschrittenes Nierenzellkarzinom mTOR-Inhibitor Everolimus schließt wesentliche Therapielücke

PharmaForum in ONKoLogie 32 | 12 | 09

(c) 2009 by S. Karger Verlag für Medizin

und Naturwissenschaften $\mathrm{GmbH}$

Wilhelmstraße 20A

79098 Freiburg, Deutschland

Mit freundlicher Unterstützung durch Novartis Pharma GmbH.

Der Verlag und die Herausgeber der Zeitschrift übernehmen keine Verantwortung für diese Rubrik. 


\section{«Proof of Concept» für die mTOR-Inhibition in der Hämatologie Temsirolimus $\left(\right.$ Torisel $^{\circledR}$ ) verlängert Überleben beim Mantelzell-Lymphom}

Der intravenöse mTOR-Inhibitor Temsirolimus (Torisel ${ }^{\circledR}$ ) hat im August 2009 als erster Wirkstoff die Zulassung speziell für die Behandlung des rezidivierten und/oder refraktären Mantelzell-Lymphoms (MCL) durch die Europäische Kommission erhalten. Dies ist die erste grundlegende Erweiterung des therapeutischen Spektrums seit der Zulassung monoklonaler Antikörper im Jahr 1998. Grundlage sind Daten der bisher größten Phase-III-Studie zum MCL, in der das progressionsfreie Überleben von MCL-Patienten signifikant um $153 \%$ gegenüber der Investigator's Choice Therapy verlängert werden konnte.

Das Mantelzell-Lymphom (MCL) ist ein klinisch häufig aggressiv verlaufendes NonHodgkin-Lymphom (NHL) der B-Zellen und weist von diesen die schlechteste Prognose auf. Die Erkrankung tritt in der Regel in einem medianen Alter von 54-68 Jahren auf, die jährliche Inzidenz wird mit 2-3 Neuerkrankungen pro 100000 Einwohner angegeben. Als Standard in frühen Therapielinien gilt die Chemotherapie in Kombination mit Rituximab. Durch medikamentöse Therapieoptionen kann jedoch meist nur eine zeitweilige Remission erreicht werden; bei einem Großteil der Patienten wird ein Rezidiv beobachtet.

Mit der Zulassung von Temsirolimus hat sich das therapeutische Spektrum nun entscheidend erweitert: "Vor dem Hintergrund des stark vorbehandelten Patientenkollektivs hebt sich das Resultat der Behandlung mit Temsirolimus erfreulich $\mathrm{ab}$ und stellt eine echte Verbesserung der therapeutischen Optionen für diese Patienten dar», sagte Priv.-Doz. Dr. Georg Hess vom Universitätsklinikum Mainz, Präsentator und Erstautor der Zulassungsstudie, auf der gemeinsamen Jahrestagung der Deutschen Gesellschaft für Hämatologie und Onkologie e.V. (DGHO).

Der Ausgangspunkt für die Wirksamkeit von Temsirolimus beim fortgeschrittenen Mantelzell-Lymphom ergibt sich durch die Pathophysiologie des seltenen Lymphoms: Das MCL ist durch die Chromosomentranslokation $\mathrm{t}(11 ; 14) \mathrm{q}(13 ; 32)$ gekennzeichnet, die zu einer Überexpression von Cyclin D1 führt, einem zentralen Protein der Zellzyklusprogression. Dieses ist von mTOR abhängig. Durch den Einsatz von Temsirolimus wird mTOR inhibiert und dadurch weniger Cyclin D1 produziert.

\section{Progressionsfreies Überleben signifikant verbessert}

Zwei Patientengruppen erhielten in der PhaseIII-Studie Temsirolimus zunächst wöchentlich als Induktionstherapie über 3 Wochen mit $175 \mathrm{mg}$, gefolgt von entweder $75 \mathrm{mg}$ (Arm 1) oder 25 mg (Arm 2) Temsirolimus wöchentlich. Zur Behandlung der Teilnehmer in der Kontrollgruppe (Arm 3) wählten die behandelnden Ärzte eine Chemotherapie aus vorgegebenen Monotherapien aus, am häufigsten Gemcitabin i.v. (42\% der Patienten) oder Fludarabin i.v. (26\%).

\section{Diagnose des Mantelzell-Lymphoms schwierig}

Die Diagnose eines Mantelzell-Lymphoms wird durch insgesamt unspezifische Symptome erschwert: So haben etwas weniger als die Hälfte der Patienten allgemeine Krankheitszeichen wie Nachtschweiß und Gewichtsverlust, gelegentlich auch Fieber.

Selten ist zudem ein rasches Lymphknotenwachstum mit einer deutlichen Zunahme der Größe innerhalb von 3 Monaten vor Diagnosestellung, was eine schnellere Entdeckung der Erkrankung erleichtern würde. Zudem klagt nur ein Fünftel der Patienten über meist lymphombedingte Schmerzen sowie Folgen einer Anämie und/oder Thrombozytopenie. Die Folge ist, dass ein MCL oft lange Zeit unentdeckt bleibt und erst in den fortgeschrittenen Stadien III oder IV erkannt wird [2]. Für die Einteilung von Non-Hodgkin-Lymphomen wurde im Jahr 1971 die Ann-Arbor-Klassifikation erarbeitet, die 4 unterschiedliche Stadien berücksichtigt $[3,4]$ :

Stadium I: $\quad$ Befall einer einzigen Lymphknotenregion $(\mathrm{N})$ oder Befall einer einzigen Lymphknotenregion mit Übergriff auf benachbartes extralymphatisches Gewebe oder einzelner, lokalisierter Herd in einem extralymphatischen Organ $(\mathrm{E})$

Stadium II: $\quad$ Befall von 2 oder mehr Lymphknotenregionen auf der gleichen Seite des Zwerchfells $(\mathrm{N})$ oder lokalisierter Befall eines extralymphatischen Gewebes und einer oder mehrerer Lymphknotenregionen auf der gleichen Seite des Zwerchfells $(\mathrm{E})$

Stadium III: Befall von Lymphknotenregionen beidseits des Zwerchfells plus/minus Milzbefall oder Befall von Lymphknotenregionen beidseits des Zwerchfells plus/ minus Milzbefall zusätzlich zu lokalisiertem Befall extralymphatischen Gewebes

Stadium IV: Nichtlokalisierter, diffuser oder disseminierter Befall eines oder mehrerer extralymphatischer Organe oder Gewebe, mit oder ohne Befall des lymphatischen Systems

$\mathrm{N}=$ nodal, $\mathrm{E}=$ extranodal 


\section{PharmaForum}

\section{Günstiges Sicherheitsprofil unter Temsirolimus-Therapie}

Insgesamt waren die unerwünschten Ereignisse klinisch gut beherrschbar. Die Nebenwirkungen der Schweregrade 3 und 4, die sehr häufig $(\geq 10 \%)$ vorkamen, waren: Thrombozytopenie (59\%), Anämie (20\%), Neutropenie (15\%) und Asthenie (13\%).

Die für das MCL geprüften Dosierungen des mTOR-Inhibitors basieren auf den Ergebnissen einer Phase-II-Studie und unterscheiden sich grundlegend von der Anwendung beim fortgeschrittenen Nierenzellkarzinom, für das die Empfehlung bei $25 \mathrm{mg}$ Temsirolimus pro Woche liegt.

\section{Intravenöse Therapie als Grundlage für Wirksamkeit und Sicherheit}

Als Grundlage für die gute Wirksamkeit und Sicherheit von Temsirolimus beim MCL sowie dem fortgeschrittenen Nierenchung des Wirkstoffs: Es wird dadurch eine hohe initiale Konzentration $\left(\mathrm{C}_{\max }\right)$ im Blutplasma erzielt, so dass der Wirkstoff schnell ins Gewebe gelangen und dort eine starke antitumorale Wirkung entfalten kann. Das heißt, dass mTOR und die nachgelagerten Signalwege langfristig gehemmt werden, was über die Reduzierung der S6-KinaseAktivität messbar ist. Die intravenöse Gabe hat jedoch gleichzeitig den Vorteil, dass die Konzentration von Temsirolimus im Blutplasma nach der Applikation schnell wieder abfällt, wodurch sich die T-LymphozytenFunktion innerhalb von 24 Stunden wieder normalisiert [5]. Eine langfristige Hemzellkarzinom gilt die intravenöse Verabrei-

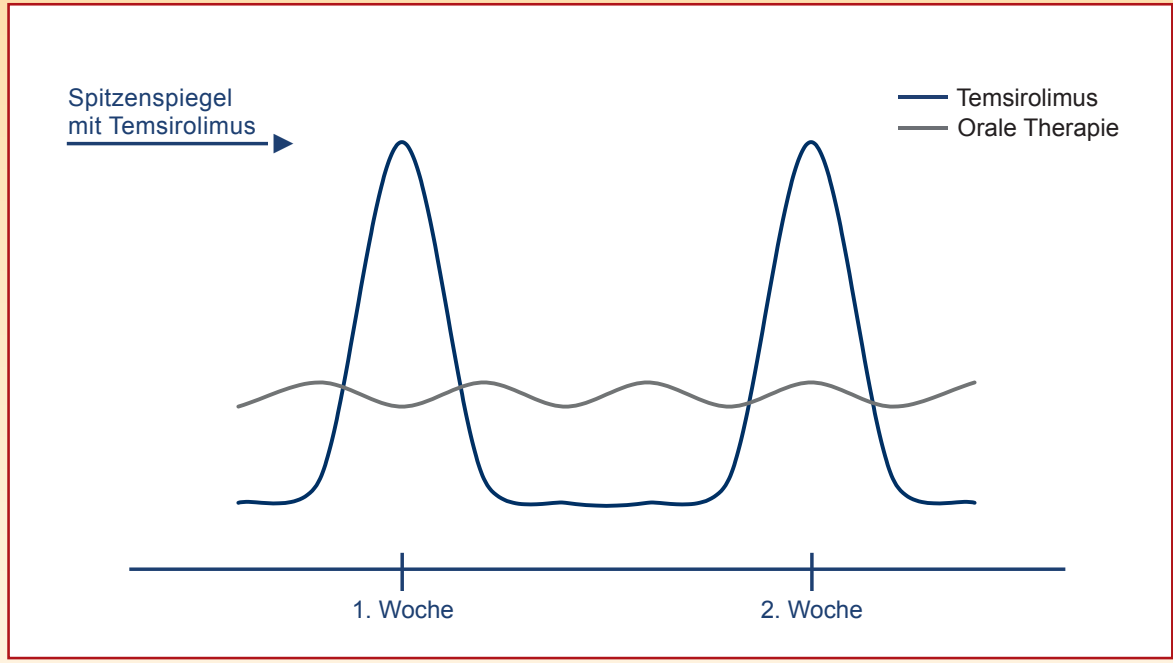

Abb. 2. Die intravenöse Therapie mit Temsirolimus führt zu hohen Spitzenspiegeln und einer langfristigen Hemmung von mTOR, messbar über die S6-Kinase-Aktivität. mung dieser zentralen Immunzellen, wie sie eine tägliche orale Gabe zur Folge hätte, kann dadurch vermieden werden (Abb. 2).

\section{Potenzial der mTOR-Inhibition in der Onkologie und Hämatologie}

Die Zulassungserweiterung hat den Stellenwert von Temsirolimus erneut bestätigt und gezeigt, dass das Wirkprinzip der intravenösen mTOR-Inhibition nicht nur bei soliden, sondern auch bei hämatologischen Tumorentitäten von Bedeutung ist. Bereits seit dem 19. November 2007 ist der Wirkstoff zur Therapie des fortgeschrittenen Nierenzellkarzinoms bei Patienten, die mindestens 3 von 6 prognostischen Risikofaktoren aufweisen, zu-

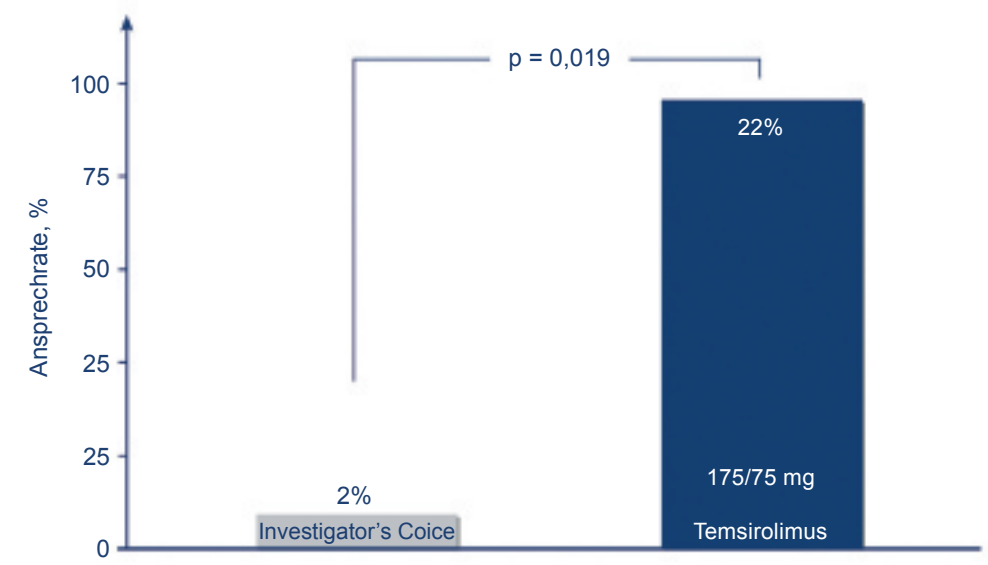

Abb. 1. Temsirolimus erhöht die Ansprechrate gegenüber Investigator's Choice signifikant [1]. gelassen; seit März 2006 besitzt er den Status als «Orphan Drug» für diese Indikation, für das MCL seit November 2006. «Ich bin davon überzeugt, dass Temsirolimus auch bei weiteren Indikationen in Zukunft eine wichtige Rolle spielen wird, da mTOR ein zentrales Protein des Zellzyklus darstellt», erläutert Professor Martin Dreyling vom Universitätsklinikum München, Leiter der «German Low Grade Lymphoma Study Group» (GLSG).

\section{Referenzen}

1 Hess $\mathrm{G}$ et al.: Temsirolimus for the treatment of patients with relapsed or refractory mantle cell lymphoma: supportive efficacy analyses from the phase 3 study. EHA 2009; \#0973.

2 Lenz G et al.: Mantle cell lymphoma: established therapeutic options and future directions. Ann Hematol 2004;83:71-77.

3 Carbone PP et al.: Report of the Committee on Hodgkin's Disease Staging Classification. Cancer Res 1971:31:1860-1861.

4 Rosenberg SA et al.: Report of the Committee on Hodgkin's Disease Staging Procedures. Cancer Res 1971;31:1862-1863.

5 Hidaloo M et al: A Phase I and Pharmacokinetic Study of Temsirolimus (CCI-779) Administered Intravenously Daily for 5 Days Every 2 Weeks to Patients with Advanced Cancer. Clin Cancer Res 2006;12(19):5755.

Impressum

"Proof of Concept" für die mTOR-Inhibition in der Hämatologie

Temsirolimus (Torisel ${ }^{\circledR}$ ) verlängert Überleben beim Mantelzell-Lymphom

PharmaForum in ONKologie 32 | 12 | 09

(c) 2009 by S. Karger Verlag für Medizin und Naturwissenschaften $\mathrm{GmbH}$

Wilhelmstraße 20A

79098 Freiburg, Deutschland

Mit freundlicher Unterstützung durch Wyeth Pharma GmbH.

Der Verlag und die Herausgeber der Zeitschrift übernehmen keine Verantwortung für diese Rubrik. 


\section{Expanded-Access-Programm bei mRCC:}

\section{Sunitinib auch in der klinischen Praxis effektiv und verträglich}

Sunitinib (Sutent $\left.{ }^{\circledR}\right)$ ist ein Standard of Care beim fortgeschrittenen/metastasierten Nierenzellkarzinom (mRCC). Die Evidenzbasis der Phase-III-Studie wird nun durch Erfahrungen aus dem Praxisalltag, auf Basis eines der weltweit größten Expanded-Access-Programme beim mRCC ergänzt. Wir sprachen mit Studienleiter Professor Martin Gore vom Royal Marsden Hospital in London auf dem ECCO/ ESMO 2009 in Berlin über die Ergebnisse des Sunitinib-Expanded-Access-Programms (EAP).
Herr Professor Gore, welche Ziele hatte das Sunitinib-Expanded-Access-Programm?

Das Expanded-Access-Programm hatte vor allem das Ziel, Sunitinib einem erweiterten Patientenkollektiv zukommen zu lassen und weitere Erfahrungen im Therapiealltag zu gewinnen. Daher konnten auch Patienten eingeschlossen werden, die aufgrund ihrer länderspezifischen Zulassungssituation bisher keinen Zugang zu einer Therapie mit Sunitinib hatten oder die an der Zulassungsstudie nicht teilnehmen konnten. Dies betraf Patienten mit eher ungünstiger Prognose oder mit Komorbiditäten.

Welche Patienten wurden innerhalb des EAP mit Sunitinib behandelt?

Das EAP war eine sehr große internationale Studie mit insgesamt 4564 Patienten in 52 Ländern. Die Einschlusskriterien waren sehr breit gefasst. So haben wir auch mRCC-Patienten mit ungünstiger Prognose aufgenommen, beispielsweise aufgrund von Hirnmetastasen, nicht klarzelligem RCC oder einem ECOG-PerformanceStatus $\geq 2$. Diese schwierig zu behandelnden Subgruppen haben uns besonders interessiert. Im Vordergrund standen dabei Fragen zur Sicherheit und Langzeitverträglichkeit im klinischen Alltag. Gleichzeitig wollten wir Informationen über die Wirksamkeit in dieser heterogenen Patientengruppe gewinnen, die sowohl Erstlinien- als auch Zweitlinienpatienten einschloss.

Was sind die wichtigsten Ergebnisse des EAP?

Die Ergebnisse des EAP weisen in die gleiche Richtung wie die Phase-III-Daten. So betrug das progressionsfreie Überleben in der Gesamtpopulation 10,9 Monate und in den Subgruppen zwischen 5,1 und 11,3 Monaten (Tab. 1). Die beobachteten Nebenwirkungen waren in der Regel leicht bis moderat. Neue oder kumulative Toxizitäten sind nicht aufgetreten. Besonders bemerkenswert ist, dass auch Patienten mit ungünstiger Prognose aufgrund von Hirnmetastasen oder schlechtem Allgemeinzustand von Sunitinib profitieren konnten. Diese Subgruppen unterschieden sich auch hinsichtlich der Verträglichkeit von Sunitinib nicht von der Gesamtpopulation. Die objektive Ansprechrate lag bei $12 \%$ für $\mathrm{Pa}$ tienten mit Hirnmetastasen, 9\% für Patienten mit ECOG-PS $\geq 2,9 \%$ für Patienten mit nichtklarzelliger Tumorhistologie und $17 \%$ für Patienten, die älter als 65 Jahre waren. Der klinische Nutzen betrug in der Gesamtpopulation $76 \%$, in den schwer zu behandelnden Subgruppen zwischen $61 \%$ und $77 \%$. Das Gesamtüberleben lag über alle Gruppen hinweg bei 18,4 Monaten. Dabei muss beachtet werden, dass über zwei Drittel der Patienten Sunitinib als Zweitlinientherapie erhalten haben.

Welche Schlussfolgerungen lassen sich auf Basis des EAP für die Therapie des mRCC mit Sunitinib ziehen?

In der First-line-Therapie des mRCC ist Sunitinib heute ein Referenzstandard. Die Daten aus dem EAP stützen die bekannten Ergebnisse aus der Phase-III-Studie. Das EAP zeigt, dass Sunitinib auch in einem breiten Patientenkollektiv wirksam ist. Die Verträglichkeit war in allen Subgruppen vergleichbar mit der

\begin{tabular}{|lccccc|}
\hline & Gesamt & $\geq 65$ Jahre & $\begin{array}{c}\text { Nicht kleinzellige } \\
\text { Histologie }\end{array}$ & $\begin{array}{c}\text { Hirn- } \\
\text { metastasen }\end{array}$ & $\begin{array}{c}\text { ECOG- } \\
\text { PS } \geq 2\end{array}$ \\
\hline Patientenzahl, $n$ & 4349 & 1056 & 437 & 213 & 319 \\
\hline PFS, Monate & 10,9 & $\begin{array}{c}11,3 \\
(10,7-12,3)\end{array}$ & $\begin{array}{c}7,8 \\
(6,3-8,3)\end{array}$ & $\begin{array}{c}5,6 \\
(5,2-6,1)\end{array}$ & $\begin{array}{c}5,1 \\
(4,2-5,2)\end{array}$ \\
\hline $\begin{array}{l}\text { Klinischer Nutzen } \\
\text { (CR + PR + SD) }\end{array}$ & $\begin{array}{c}76 \% \\
(\mathrm{n}=3464)\end{array}$ & $77 \%$ & $68 \%$ & $64 \%$ & $61 \%$ \\
\hline \multicolumn{4}{l}{ PFS: progressionsfreies Überleben; CR: komplette Remission; PR: partielle Remission; SD: Stable Disease } \\
\hline
\end{tabular}

Tab. 1. Effektivität im Sunitinib-Expanded-Access-Programm: Gesamtpopulation und Subgruppen [1]

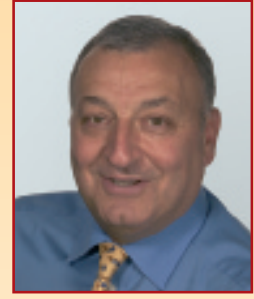

Interview mit

Professor Martin Gore, Medical Director des Royal Marsden Hospitals in London und Professor für Krebsmedizin am Institute of Cancer Research in London

Gesamtpopulation. Auch die Effektivitätsdaten und die Daten zur Sicherheit in der Anwendung waren konsistent mit den Ergebnissen der Zulassungsstudie. Was die Subgruppen betrifft, lässt sich sagen, dass auch ältere Patienten, Patienten mit mRCC und Hirnmetastasen, nicht klarzelliger Histologie und ECOG-PS $\geq 2$ von einer Therapie mit Sunitinib profitieren können. Dies ist eine wichtige Botschaft in Bezug auf die manchmal schwierige Therapiesituation des klinischen Alltags.

In Ihrem Vortrag auf dem ECCO/ESMO haben Sie betont, dass das Therapiemanagement bei mRCC eine wichtige Rolle spielt. Können Sie uns erklären, weshalb und was dabei zu beachten ist?

Damit die Patienten einen größtmöglichen Nutzen von Sunitinib haben, sind drei Punkte besonders wichtig: Sie müssen - ausgehend von der Standarddosierung von $50 \mathrm{mg}$ täglich - die optimale individuell abgestimmte Dosis erhalten, und die Therapie muss bis zum gesicherten Krankheitsprogress fortgeführt werden. Von großer Bedeutung ist auch ein proaktives Therapiemanagement, das den Patienten von Anfang an in den Therapieprozess einbezieht. Ziel ist es, den Patienten möglichst lange und mit der optimalen Dosis zu behandeln. Nur so können wir einen maximalen Therapieerfolg gewährleisten.

Anne Krampe-Scheidler, Berlin

\section{Referenz}

1 Gore ME et al.: Lancet Oncol 2009;10(8):757-63.

\section{Impressum}

Expanded-Access-Programm bei mRCC: Sunitinib auch in der klinischen Praxis effektiv und verträglich

PharmaForum in ONKoLogie 32 | 12 | 09

(c) 2009 by S. Karger Verlag für Medizin und Naturwissenschaften $\mathrm{GmbH}$

Wilhelmstr. 20A

79098 Freiburg, Deutschland

Mit freundlicher Unterstützung durch Pfizer Pharma GmbH.

Der Verlag und die Herausgeber der Zeitschrift übernehmen keine Verantwortung für diese Rubrik. 